\title{
La promoción de la ciudadanía como clave de una intervención inclusiva y participativa
}

\author{
Encouragement of the Public as the Key to an Inclusive \\ and Participatory Intervention
}

\author{
OEISM $^{1}$ \\ Observatorio sobre la Exclusión y los Procesos de Inclusión en la Comunidad de Madrid \\ observatorioinclusion@gmail.com
}
Estar organizados nos hace más fuertes y nuestras acciones más efectivas Participante taller de ciudadanía, febrero 2010

Recibido: 09/02/2012

Revisado: 29/02/2012

Aceptado: 14/03/2012

Disponible on line: 03/08/2012

\begin{abstract}
Resumen
En los últimos años vuelve a resurgir con fuerza el debate sobre la desigualdad: la no reducción de los índices de pobreza, el aumento de la distancia entre ricos y pobres, el distanciamiento de las grandes estructuras de poder y decisión de los problemas de los ciudadanos y la aparición de voces críticas que exigen el cumplimiento de los Derechos Humanos en el marco de una sociedad global en crisis. Partiendo de este contexto y de la noción de ciudadanía, que implica el acceso y desarrollo de un conjunto de derechos y deberes para la satisfacción de las necesidades humanas y la vida en comunidad, este artículo relata la experiencia de intervención del Observatorio sobre la exclusión y los procesos de inclusión en la Comunidad de Madrid (OEISM) con colectivos en situación de exclusión orientada al desarrollo de sus vínculos comunitarios y la participación social plena.
\end{abstract}

Palabras clave: exclusión, ciudadanía, participación, necesidades humanas, derechos sociales.

\begin{abstract}
In recent years, the debate on inequality has vigorously resurfaced: the lack of reduction in poverty rates, the increase in the gap between rich and poor, the distancing of the large power and decision-making structures, and the emergence of critical voices demanding the implementation of human rights in the context of a global society in crisis. Within this context and based on the notion of citizenship, which implies access and development of a set of rights and duties for the satisfaction of human needs and community life, this article describes the experience the «Observatory on Exclusion and Processes of Inclusion in the Community of Madrid» (OEISM) with groups in situations of exclusion aimed at the development of their community ties and full social participation.
\end{abstract}

Key words: exclusion, citizenship, participation, human needs, social rights.

Referencia normalizada: OEISM (2012): «La promoción de la ciudadanía como clave de una intervención inclusiva y participativa». Cuadernos de Trabajo Social, 25(2): 393-402.

Sumario: 1. La experiencia de las comisiones ciudadanas en el marco del proyecto Observatorio de la exclusión social y los procesos de inclusión en la Comunidad de Madrid. 2. Los talleres de ciudadanía como estrategia para la constitución de las comisiones ciudadanas. 3. Posicionándonos frente a la exclusión. 4. Barreras y dificultades de acceso y ejercicio de los derechos de ciudadanía. 5. Recursos con los que contamos para la consecución de derechos. 6. Recursos con los que contamos para la consecución de derechos. 7. Propuestas de trabajo. 8. Conclusiones, aprendizajes y retos de futuro. 9. Referencias bibliográficas.

${ }^{1}$ Los miembros del Grupo de Análisis sobre Exclusión y Promoción de la Ciudadanía del Observatorio que hn elaborado el texto son los siguientes: Myriam Alonso (Asociación INTRESS); Marinete Alves (Asociación OEISM); Mariano Barba (Fundación Atenea); Manuel Basagoiti y Paloma Bru (Asociación REDES), Ana Carbajo (Asociación CRISOL); Elisa Corral y Azucena Magdaleno (Asociación Candelita); Rocío García (Fundación Secretariado Gitano); Lucila Maquieira (Asociación DEIS) y Margarita Navarro (UCM). 
1. La experiencia de las comisiones ciudadanas en el marco del proyecto Observatorio de la exclusión social y los procesos de inclusión en la Comunidad de Madrid

Creado en el año 2007, el Observatorio sobre la Exclusión social y los Procesos de inclusión en la Comunidad de Madrid (OEISM, www. observatorioinclusión.org) es un proyecto en el que participan diversas instituciones académicas (Universidad Complutense de Madrid Escuela de Trabajo Social) y entidades de acción social, profesores universitarios y expertos en el ámbito de la lucha contra la pobreza y la exclusión social. Pretende ser un espacio y una herramienta que posibilite la investigación, reflexión y encuentro entre profesionales, entidades e instituciones vinculadas a la intervención social y tiene como objeto la promoción del intercambio de información, conocimiento y propuestas metodológicas que contribuyan a la mejora de los procesos de inserción de las personas en riesgo o situación de exclusión.

En el marco del Observatorio se cubren asimismo diferentes objetivos de actuación a través de grupos de trabajo autónomos, en su forma de organización y regulación interna, metodología y contenidos temáticos que abordan, pero interconectados a través de la asamblea, como órgano de participación y toma de decisiones democrática del mismo.

Dentro de la estructura del Observatorio, el Grupo 1 de análisis de la exclusión aborda tanto la tarea analítica de los procesos de exclusión, como la de dinamización de una comisión ciudadana, un espacio de participación en el que se quiere dar presencia y voz a los propios ciudadanos que «vivencian y representan el hecho social de la exclusión». El Grupo está formado por profesionales de distintas entidades sociales que desarrollan proyectos e iniciativas de inserción sociolaboral en el ámbito de la Comunidad Autónoma de Madrid: Asociación Candelita, Asociación CEPA, Asociación Crisol, Asociación DEIS, Asociación La Kalle, Asociación REDES, Fundación Secretariado Gitano, INTRESS, Fundación Atenea y Asociación OEISM

Desde sus inicios, en 2008, el debate del grupo se ha centrado fundamentalmente en dos ejes temáticos: la exclusión y su medición y la ciudadanía y su promoción. Trabajar sobre estos dos ejes ha supuesto un proceso continuo de reflexión y acción, avanzando en análisis conjuntos sobre conceptos, factores e indicadores y sobre las metodologías y estrategias más adecuadas para llegar a cubrir nuestros objetivos desde una lógica cooperativa y participativa.

\section{Los talleres de ciudadanía como estrate- gia para la constitución de las comisiones ciudadanas}

\subsection{Introducción metodológica}

Si buscamos una referencia metodológica al trabajo grupal que iniciamos en el año 2008 no podemos centrarnos en una sola, sino en la pluralidad de influencias que, de una forma más o menos explícita y planificada, han guiado nuestra tarea. La promoción de espacios de ciudadanía con personas que hallan importantes barreras para acceder a ella en condiciones de igualdad y equidad con el resto de los ciudadanos, nos plantea un reto importante: la necesidad de idear una metodología que permita, por un lado, conocer más a fondo la realidad social de estas personas (investigación), y por el otro, abrir un proceso de ciclo largo para que este conocimiento de la realidad adquiera una dimensión participativa, contando con los propios sujetos cuando se vayan a analizar las causas de su situación (participación) y, en tercer lugar, avanzar en la construcción colectiva de propuestas de transformación y mejora, de su propia realidad y de las personas que se encuentran en su misma situación (acción).

Estos tres ejes son la esencia de la Investigación-Acción Participativa, que propone el análisis de la realidad como una forma de conocimiento y sensibilización de la propia población (Rubio y Varas, 1997). Ésta se transforma, en el proceso, en sujeto activo y protagonista de su propio cambio y con ello alcanza mayores cotas de protagonismo activo para la mejora de su entorno y realidad más inmediata: ámbitos de vida cotidiana, espacios de relación comunitaria, barrio, distrito, municipio, etc. (Basagoiti, Bru. y Lorenzana, 2001).

Sin embargo, la Investigación-Acción Participativa no sólo es una metodología para investigar, sino también se descubre en su proceso como una auténtica herramienta de intervención social. Según Pérez Serrano (1990) puede considerarse como un proceso que lleva a cabo una determinada comunidad (o colectivo, en 
nuestro caso), para llegar a un conocimiento más profundo de sus problemas y tratar de solucionarlos, intentando implicar a todos los miembros del grupo en el mismo. Nos permite profundizar en el conocimiento de la realidad social, de las circunstancias concretas de cada grupo social y del entorno en el que vive (en muchas ocasiones con un índice importante de vulnerabilidad) que serán quienes determinen de qué forma se va a hacer la investigación y con qué medios se cuenta para ello. Sin embargo existen algunos criterios básicos a los que hemos atendido:

- Se trata de una población con un nivel elevado de vulnerabilidad social, lo que exige un planteamiento del trabajo en clave de proceso de ciclo largo y es necesario adaptar las técnicas a emplear a las circunstancias de cada grupo social.

- Nos hemos marcado unos objetivos, como la puesta en marcha de espacios de promoción de la ciudadanía y la apertura de procesos de mejora de la calidad de vida de personas en situación de vulnerabilidad social.

- En el proceso nos encontramos con algunos problemas concretos que debemos ir solucionando, como ciertas resistencias, amenazas, cambios en las situaciones personales de las personas de los diferentes grupos, etc.

- Nos abrimos a una pluralidad de perspectivas y puntos de vista que tiene la gente ante los problemas y recogemos las propuestas que hayan salido a través de la participación, para poder debatirlas y negociar sobre ellas a todos los niveles (en los grupos, entre los técnicos, entre todos). Las personas implicadas tienen protagonismo, aportan propuestas que marcarán la acción en el futuro.

- Cuando se ponen en marcha las líneas de actuación emergen otros problemas para los que habrá que buscar nuevas soluciones; el proceso se retroalimenta.

- En todo momento hemos respetado la libertad del individuo y su decisión de participar o no en el espacio de debate propuesto, así como hemos preservado el anonimato y el derecho a que le sea devuelta toda la información extraída —o recogida - a lo largo del proceso.

- A cada taller le ha precedido un encuentro con los profesionales de la entidad para coordinar los contenidos y estrategias de abordaje de los temas a tratar.
- Se ha procurado mantener, en definitiva, la transparencia, la claridad de los objetivos y la limitación de otras expectativas que los participantes pudieran plantear (consecución de un empleo, alojamiento, prestación monetaria, etc.).

\subsection{Planteamiento y desarrollo de los talle- res de ciudadanía}

Con estas bases metodológicas y éticas hemos puesto en marcha los primeros talleres de ciudadanía. Se plantearon como una reunión grupal con personas beneficiarias de los programas de empleo, gestionados por las entidades que participan en el Observatorio de la exclusión social y los procesos de inclusión en la Comunidad de Madrid. En ellos se trabajó fundamentalmente sobre el significado de ser ciudadano, el conocimiento de los derechos por los sujetos en situación de exclusión, las barreras de acceso y ejercicio de los mismos y las formas de superarlas.

El principal objetivo era recoger la voz de los sujetos a los que se dirigen las políticas sociales y los proyectos de inserción, pero a los que rara vez se escucha en el momento en que se están diseñando o cuando se implantan (¿Dónde están los excluidos?, ¿qué piensan?, ¿qué sienten? y ¿qué quieren?).

Un segundo objetivo era validar y contrastar las reflexiones y análisis del Grupo 1 del $O b$ servatorio, conformado por responsables y técnicos profesionales de los recursos y programas de integración (proyectos de orientación sociolaboral, formación ocupacional, servicios de intermediación laboral, acompañamiento social, itinerarios integrados de empleo y empresas de inserción).

El planteamiento concreto de cada taller dependía del perfil de los participantes, pero se acordó buscar una dinámica de grupo que facilitara la participación activa y que la sesión tuviera una duración determinada (una hora y media). En los primeros nueve talleres participaron 113 personas con los siguientes perfiles:

- Mujeres perceptoras de renta mínima de inserción.

- Hombres perceptores de renta mínima de inserción.

- Jóvenes en riesgo de exclusión.

- Población de etnia gitana. 
- Personas con enfermedad mental en proceso de rehabilitación laboral

- Jóvenes de origen extranjero.

- Mujeres de origen extranjero.

- Personas en tratamiento de drogodependencias.

- Personas en situación de exclusión residencial.

Los talleres se han desarrollado con una metodología abierta de investigación-acción-participación. Este último aspecto nos ha llevado a realizar dos encuentros para cada uno de ellos:

- Un primer taller de debate sobre derechos de ciudadanía (nueve talleres)

- Un segundo taller de devolución de los resultados generales y recogida de propuestas (cuatro).

A largo plazo, la finalidad de los talleres de ciudadanía es articular un espacio de participación dentro del Observatorio de exclusión, que hemos llamado Comisión Ciudadana. Su sentido, dentro de la estructura y metodología del Observatorio, es aportarle un valor añadido, el de la participación directa y permanente de la ciudadanía, concretamente de las personas que se encuentran en procesos de inserción sociolaboral en diferentes instituciones y organizaciones sociales. Esta Comisión pretende ser un grupo de contraste de los análisis y temáticas que se están trabajando en otros grupos de trabajo del Observatorio (ciudadanía, derechos humanos, factores e indicadores de exclusión, etc.). A su vez, las personas participantes en el grupo, como beneficiarias directos de políticas públicas de inserción, analizarán los diferentes temas propuestos partiendo de su propia experiencia y de la de otras personas cercanas a ellas. Este punto también se convierte en un momento de reflexión y autodiagnóstico sobre su entorno y de contraste de su propia experiencia con la de otras personas en situación similar.

\subsection{Devolución y continuidad del proceso}

Tras los talleres iniciamos una fase de devolución a todos los grupos de lo recogido. En ese el momento cada colectivo tomaba conciencia de los problemas desde una dimensión más compartida con otras personas afectadas por otros factores, en principio diferentes. Gracias a este paso, fueron los propios grupos los que propusieron encontrarse y conocerse de una forma distendida y en la que pudieran compartir sus preocupaciones y también sus propuestas de mejora, para hacerlas de todos.

Iniciamos entonces una nueva etapa en el que cada grupo debía trabajar con el apoyo de su recurso-entidad para presentar al resto de grupos sus reflexiones en torno a la idea de ciudadanía. Con el fruto de este trabajo se han realizado ya dos Encuentros de ciudadanía (en abril de 2010 y junio de 2011), celebrados en el Centro social comunitario Casino de la Reina de Madrid, con los colectivos que participaron en los talleres y en las comisiones ciudadanas (personas con enfermedad mental, personas sin hogar, personas de la comunidad gitana, mujeres perceptoras de la RMI, personas en rehabilitación por drogodependencias, jóvenes con dificultades de acceso al empleo, inmigrantes, etc.), quienes mostraron su visión de las dificultades a las que se enfrentan a diario para ejercer sus derechos, a través de diferentes soportes creativos (radio libre y comunitaria, audiovisuales, paneles, canción, poesía, etc.). En estos espacios, los grupos tienen la oportunidad de conocerse, intercambiar sus puntos de vista y reflexiones y construir conjuntamente propuestas.

Estos Encuentros han ido acompañados de otros espacios de carácter más formal en los que han podido hacer llegar propuestas a técnicos y políticos (Jornadas que el Observatorio celebró en la Casa Encendida, en junio de 2010) y en los que han podido compartir su testimonio y experiencia vital con profesionales y estudiantes (como el Encuentro organizado en la Escuela de Trabajo Social de la Universidad Complutense de Madrid, en noviembre de 2011 con motivo de la Semana de la Ciencia).

Paralelamente a estas actividades, las comisiones ciudadanas, las entidades participantes y el propio Observatorio han editado materiales de sensibilización y didácticos con diferentes soportes: un manual metodológico enfocado a la realidad de las personas con enfermedad mental crónica, vídeos sobre la realidad de los diferentes colectivos, paneles, informes, blogs, página web, etc. La continuidad y el refuerzo de este proceso, que ha alcanzado cada vez mayores cotas de participación, apunta hacia la constitución de un nuevo espacio asambleario en el que confluyan personas de diferentes barrios y municipios de Madrid para que se oiga su voz y re- 
clamar su derecho a formar parte de la sociedad en condiciones dignas de vivienda, empleo, educación, salud y garantía de ingresos.

\section{Posicionándonos frente a la exclusión}

Una gran parte de nuestro quehacer se ha centrado en analizar los vínculos entre dos conceptos fundamentales: el de exclusión y el de ciudadanía. Asociarlos ambos implica resaltar que la existencia del primero para muchos de estos colectivos comporta limitaciones para el desarrollo del segundo y la negación de aquellos derechos que posibilitan la ciudadanía para muchas personas, que hace crecer el espectro de situaciones de exclusión social. Según Martínez de Pisón (1997):

El Estado debe intervenir y responsabilizarse de la situación material de los ciudadanos y procurar la satisfacción de sus necesidades. A diferencia de los derechos civiles y políticos, los derechos sociales crean obligaciones positivas, en la medida en que son sólo realizables a través de la acción social del Estado.

Podemos entender entonces la ciudadanía como el derecho a la integración en la sociedad y el deber de ésta de integrar a su población más vulnerable. Ser ciudadano, por lo tanto, es serlo con todos los derechos integrados, pero la exclusión desintegra los comportamientos imprescindibles para la asunción de esos derechos. Así, debemos concebir la exclusión como un disolvente ciudadano y, como tal, como algo que socava las posibilidades de desarrollo pleno de las normas democráticas que supuestamente nos guían.

Definimos la exclusión como un concepto «complejo, multidimensional y dinámico». Como concepto dinámico, incorpora la idea de proceso variable y cambiante (no una situación estática e inmutable); y como concepto multifactorial, la interacción de diversas causas o variables de tipo social, psicológica, cultural, económica, político-legal, etc. No poner en marcha medidas integrales que aborden este problema tiene una consecuencia fundamental en las situaciones de exclusión: su crecimiento en aspectos claves para la calidad de vida, que conllevan deterioros económicos, culturales, de vivienda, de comportamientos, relacionales, etc. (Alves, Basagoiti y Bru, 2009).
Las organizaciones sociales estamos interviniendo sobre los factores que generan exclusión con las personas que participan en nuestros programas: en primer lugar, todos los grupos con los que trabajamos se ven afectados por una situación de desempleo o subempleo. La precariedad además afecta a la calidad del empleo existente. Las mujeres, los jóvenes y las personas mayores de 45 años son los que más acusan esta situación de inestabilidad y vulnerabilidad; y muchas de las personas con las que trabajamos se hallan en un escalón inferior al de la precariedad laboral, anclados en una situación de desempleo de larga duración y de imposibilidad de acceso al mercado laboral.

En los jóvenes destaca el hecho de que se han socializado en la precariedad y la mayor incidencia en su proceso de exclusión del factor educativo es el fracaso escolar. La formación es importante y cuanto más avanzada es la edad las posibilidades de aprovecharla o retomarla disminuyen. El factor económico (renta) parece clave: la escasez de recursos económicos está en el centro del problema de exclusión y de las dificultades de acceso a otros recursos de inserción. La garantía de ingresos debe ser una prioridad de la política social. Unido a la falta de empleo a la que hemos aludido antes, aunque no es el único «factor de entrada» en la situación de exclusión, contribuye a la acumulación de diferentes circunstancias y condiciones que conducen, en un determinado momento vital, a la ruptura de los vínculos sociales y a la pobreza (separaciones o divorcios, desempleo, dificultad de pago de vivienda, consumo, enfermedad, etc.). Se trata de un factor relevante, el de la inestabilidad o ruptura de las redes sociales formales e informales, por su función de articulación, de conexión entre personas y grupos, y como estructura de apoyo. Las redes también pueden estructurarse sobre lógicas incluyentes o excluyentes.

A los elementos anteriores se suma la imposibilidad de acceso a la vivienda y la mala calidad de la misma (infravivienda), como un factor que influye de manera decisiva en Madrid en agravar la exclusión y está estrechamente vinculado con otros factores, como los que llevan a la estigmatización territorial (los llamados barrios vulnerables). Finalmente, la escasez de recursos o la infradotación de los mismos, el cierre de servicios especializados de indudable 
utilidad para estos colectivos, la falta de servicios de emergencia, la saturación de los existentes, etc. son elementos de política social que sin duda cobran mayor relevancia en el actual contexto de crisis económica.

Por este motivo, en íntima relación con los factores relatados, nos parece fundamental abordar la exclusión desde la combinación de dos ejes centrales: la no satisfacción de las necesidades humanas y el incumplimiento de los derechos humanos básicos, políticos y sociales, que son en último término la garantía de acceso y ejercicio de la ciudadanía plena.

La crisis está teniendo repercusiones graves en los colectivos en situación de exclusión. No solo se refleja - como hemos apuntado- en el aumento del desempleo, la precariedad y la falta de oportunidades a causa de la fuerte competencia, sino que además se está acentuando una fuerte desconfianza hacia el sistema, un sentimiento de autoexclusión debido a las condiciones de inferioridad de estas personas con respecto al resto de la sociedad. Ante esta nueva realidad, se generaliza una respuesta social y política que tiene una doble cara: por una la vertiente estigmatizadora, culpabilizadora y exclusógena, en la que el abordaje político que se hace de esta crisis muestra unos cauces democráticos de toma de decisiones (políticas, económicas y sociales) muy alejados de las personas y aún más de aquellas a las que el sistema ha expulsado; y la otra que es más esperanzadora, por lo integradora y solidaria, sin embargo ante la desconfianza a la que hemos aludido antes, parece más bien un maquillaje para ocultar las desigualdades.

\section{Barreras y dificultades de acceso y ejer- cicio de los derechos de ciudadanía}

Los participantes en los talleres de ciudadanía identifican el término «acceso a derechos» con tres aspectos claves: la existencia y la suficiencia de los recursos y servicios dirigidos a los colectivos con mayores dificultades de inserción; la disposición de una información clara y accesible sobre los mismos y sobre los requisitos de «entrada»; y el conocimiento de los procedimientos y la adecuación de los mismos.

La población de etnia gitana, las personas afectadas por el «sin-hogarismo» y las personas de origen inmigrante son los que en mayor medida señalan la imposibilidad de ejercer unos derechos que no se tienen reconocidos por ley o a los que no se tiene acceso por falta de recursos. La imposibilidad de acceso a un empleo estable (más aún en contextos de crisis) y de calidad y la situación jurídico-legal (nacionalidad o situación regular), son dos aspectos que impiden el acceso a otro tipo de derechos: vivienda, educación, participación, etc. En ese sentido, el derecho al trabajo es prioritario por su función instrumental, igual que el derecho a la educación, en la medida en la que facilita el acceso a recursos y servicios de calidad. El derecho al descanso, al ocio, al disfrute de la cultura, a la participación o al afecto, incluso, son derechos que quedan desplazados porque prevalecen otros como el empleo o por la autoexclusión de esos ámbitos, lo que, en ocasiones, se convierten en nuevas barreras para la integración en la comunidad, el barrio o el municipio.

La discriminación por diversos motivos y el estigma social son dos barreras que afectan a determinados grupos, entre ellos, a la población gitana, a las mujeres, a las personas con enfermedad mental, a los inmigrantes y a los toxicómanos. Este factor «exclusógeno» se extiende al conjunto de la sociedad a través de mensajes estereotipados, transmitidos por los diferentes estamentos políticos, económicos, jurídicos, y difundidos por los medios de comunicación.

La inseguridad o falta de garantía jurídica es una barrera mencionada únicamente por los grupos de personas inmigrantes. Este factor limita la libertad de movimiento y el ejercicio de derechos políticos (voto), lo que incide en la negación de su condición de ciudadanía. El propio sistema público y los diferentes poderes del Estado pasan a ser considerados como obstáculos para el ejercicio de derechos por diferentes causas: falta de regulación y aplicación de las normas que se aprueban en los diferentes niveles de gobierno (central, autonómico, local), escasez de recursos públicos materiales y humanos, excesiva burocracia en los procedimientos y dificultades para tramitar las ayudas públicas.

La falta de información, acerca de derechos $\mathrm{y}$ canales de acceso y defensa son, en último término, un obstáculo para poder reclamarlos y defenderlos, y se aborda resaltando la importancia de la formación en y para el ejercicio de la ciudadanía, la participación y la organización colectiva. 


\section{Recursos con los que contamos para la con- secución de derechos}

Las personas participantes en los talleres conocen algunos canales e instrumentos de acceso a sus derechos, aunque no siempre los utilizan (es el ejemplo de los sindicatos, las asociaciones de defensa de los derechos humanos, etc.). Los recursos de apoyo con los que dicen contar son un vínculo a la vez instrumental y afectivo: sirven para satisfacer necesidades, pero en ocasiones generan dependencias. Sin embargo «conocer no basta» o no es suficiente para acceder y tener garantizados esos derechos, es condición necesaria pero no suficiente, «hay que conocer y hacer», esto es: dar el paso siguiente y alcanzar mayores cotas de participación.

Se delega en otros la responsabilidad de velar por nuestros derechos e intereses: el Estado, los gobernantes, los políticos, los empresarios, etc. De ese modo no se asume un papel más activo en la defensa de los propios derechos, como pudiera ser por otras vías como el asociacionismo o el apoyo individual a otras organizaciones sociales en aquella demandas y reclamaciones que contribuyen a incrementar el bien común. El hábito de la participación es saludable para la democracia. Tenemos el derecho y el deber de participar. En el camino hacia la consecución de nuestro desarrollo pleno como sujetos de derechos no hay atajos, pero tampoco estamos solos: las redes de colaboración y el trabajo cooperativo son uno de los caminos posibles.

\section{Oportunidades que podemos aprovechar}

La existencia de equipos de trabajo multiprofesionales permite iniciar procesos de trabajo grupales y en redes interdisciplinares, optimizar métodos y recursos de intervención y abarcar los diferentes ámbitos de relación de las personas que participan en los procesos de inserción (personales, laborales, comunitarios, sociales, etc.). En determinados grupos sociales, la mujer representa la oportunidad de cambio para todo el conjunto de la comunidad (por ejemplo en la etnia gitana), es más receptiva para entrar en procesos de formación básica y ocupacional y porque es una gran transmisora de los nuevos saberes y valores sociales en la institución de la familia. Otros impulsores de cambio son también las personas que han participado en procesos de promoción social y laboral, trabajan y se incorporan activamente en la sociedad y ejercen un papel mediador y de liderazgo hacia dentro, al mismo tiempo que proyectan hacia fuera imágenes positivas de la comunidad o grupo de referencia. Asimismo, el voluntariado activo constituye otra oportunidad para generar prácticas inclusivas y de ciudadanía, si entendemos su papel como «emprendedor social» y portador de valores de participación, compromiso, responsabilidad y justicia social.

Por su parte, los procesos de desarrollo local comunitario son también experiencias que se consideran oportunidades de integración social, por los valores de participación que promueven y por su efecto contagio e ilusionante en otros contextos territoriales. El reto consiste en aprovechar estos espacios y procesos para promover una mayor participación del conjunto de la base social y de las personas en situación de o con riesgo de exclusión con las que trabajamos. Estas experiencias y nuevas metodologías participativas son escasas o residuales, pero son interesantes por su creciente presencia y su utilidad como espacios potencialmente integradores.

Frente a estas dificultades, sobre todo en las situaciones dramáticas personales y familiares que se están desencadenando por efecto de la crisis financiera y el aumento del desempleo, podemos considerar este panorama crítico como una nueva oportunidad para recuperar las redes de relaciones sociales basadas en la solidaridad y el apoyo mutuo (redes de trueque, cooperativas de consumidores, bancos del tiempo, etc.). Asimismo la crisis nos brinda la oportunidad de revisar nuestro modelo de desarrollo, nuestros valores culturales y nuestros patrones de consumo y de relación social.

\section{Propuestas de trabajo}

7.1. ¿Cómo podemos reforzar desde las entidades los vínculos comunitarios y los canales de acceso a derechos?

Las entidades sociales que trabajamos en el ámbito de la exclusión e inclusión social debemos profundizar en el conocimiento de los derechos de los participantes de nuestros proyectos de inserción, así como facilitar información y formación acerca de los recursos de los que disponen para poder acceder a ellos (justicia gratuita, ventanillas administrativas, oficinas de atención ciudadana, órganos de participación y consul- 
ta, mecanismos de protección social sobre los que tienen derecho, etc.).

En las organizaciones sociales hemos de velar porque el trato a las personas con mayores dificultades sea respetuoso y se valore también su tiempo, sus opiniones, deseos y decisiones. Las personas con las que intervenimos son ante todo ciudadanos, sujetos de derecho, aunque tengan dificultades en diferentes áreas de su vida. Debemos profundizar en la promoción de la participación social y el asociacionismo de las personas y grupos con quienes trabajamos, así como fomentar sus relaciones sociales y los vínculos comunitarios (con otros grupos, asociaciones, redes, etc.), de cara a la consecución de su plena autonomía e integración social. Por nuestra posición y cercanía a las situaciones de vulnerabilidad social, debemos ser facilitadores de recursos y oportunidades para que los sujetos puedan hacer llegar su voz, sus demandas y sus propuestas a las instituciones y al conjunto de la sociedad.

\subsection{Criterios, estrategias y herramientas de acción}

En el Grupo 1 también estamos analizando nuestras metodologías de intervención $\mathrm{y}$, como punto de partida, nos planteamos al menos tres criterios básicos, que han de guiar nuestra acción y que pasan a ser, a su vez, los indicadores de evaluación de nuestro propio proceso de trabajo en red: sinergia (multiplicación de resultados y niveles de impacto), integralidad (enfoque multidimensional) e integración (promoción de la cohesión social). Esta estrategia relacional-comunicacional y mediadora se despliega en cuatro ámbitos de trabajo, que componen una línea continua desde los contextos micro-individuales a los contextos macro-sociales.

\section{a) En el nivel individual}

Desarrollamos un trabajo orientado al sujeto, lo que implica:

- Atención individualizada y orientación a la persona, desde un planteamiento integral y participativo.

- Integración en cualquier proceso de educación, formación, orientación, intermediación, acompañamiento, apoyo y refuerzo, etc., dirigida a la promoción de la inserción sociolaboral, a establecer estrategias de promoción de la ciu- dadanía que favorezcan el desarrollo personal, comunitario y social con igualdad de oportunidades, respeto y tolerancia.

- Búsqueda de otras herramientas aún poco exploradas en las entidades sociales que son las que incorporan la propia creatividad y el talento artístico de las personas con las que trabajamos (teatro social, danza, pintura, etc.).

\section{b) En el nivel grupal}

Trabajamos con las familias y las redes de apoyo informales del sujeto (grupos de iguales, vecinos, actuaciones en refuerzo del tejido social informal del barrio, voluntariado).

- Instrumentos: trabajo de calle, reuniones informales y asambleas de barrio, dinámicas de grupo y talleres participativos.

- Promoción de nuevos procesos formativos en el ámbito sociocultural y el ocio (cursos de monitores de tiempo libre, por ejemplo) dirigidos a los grupos con los que intervenimos, y canalizar así sus propias iniciativas de ocio, cultura, etc.

- Fomento de espacios de sensibilización social en los que estas personas sean los auténticos protagonistas.

\section{c) En el nivel comunitario}

Pensamos que una intervención de calidad para fomentar la disminución de los factores que inciden en la exclusión tiene que abordar su dimensión territorial. Las intervenciones desarrolladas en los llamados barrios vulnerables deben atender a la potenciación de capacidades personales, familiares y colectivas, como un capital necesario para enriquecer y mejorar las condiciones de vida, desde una perspectiva vinculada al rescate de los sentidos locales, y que responda a necesidades e intereses construidos colectivamente.

Así, es fundamental fomentar factores protectores y disminuir factores de riesgo de contexto asociados directa o indirectamente a situaciones de exclusión social, trabajando con ellos como agentes de su propio desarrollo. Esta forma de actuar contribuirá a la prevención de situaciones de exclusión, desde los factores de protección familiares y comunitarios, y desde la ampliación de las posibilidades de acción social frente al entorno. Habremos apuntado a fortalecer recursos y vínculos sociales, habre- 
mos fomentado el capital social, la confianza, la autonomía y la integración comunitaria.

Construir cohesión social se convierte, por lo tanto, en una fuerza de contención de los riesgos amenazadores de exclusión, especialmente de la de las personas más cercanas al deterioro económico y de comportamientos. Para ello es necesario hacer confluir las intervenciones con procesos de desarrollo locales y comunitarios, adquiriendo la coordinación en red en este marco un impacto mucho más interesante.

\section{d) En el nivel estructural}

Debemos abordar acciones de información, sensibilización, mediación, movilización social, denuncia, negociación, concertación, etc.; para lo cual hemos de dotarnos de otros recursos y medios de apoyo e incorporarnos activamente en los espacios públicos de debate, aquellos de los que disponen los medios de comunicación social, los agentes socioeconómicos (empresas, sindicatos, etc.) y la Administración local, autonómica, etc. y que se proyectan hacia la ciudadanía en general. Por otro lado, creemos que se ha profundizado poco en la medición de la calidad de los procesos de inclusión y las políticas públicas en las que estos se enmarcan. La labor fundamental que muchas ONG de acción social y de empresas de economía social desarrollan en este sentido con eficacia y eficiencia, queda mediatizada por la dinámica de las propias políticas públicas, tanto en sus contenidos como en la metodología que utilizan en su elaboración. La falta de participación del Tercer Sector en su diseño es algo que se está debatiendo desde hace tiempo y debe ser reivindicado con mayor intensidad, pero la participación debe ser demandada no sólo para el diseño sino para el seguimiento y la evaluación de las políticas. La devolución a la ciudadanía de los resultados de estas políticas es casi inexistente.

Por otro lado, ante la necesaria revisión de las políticas existentes de protección social, estamos iniciando un debate abierto sobre la eficacia de las rentas mínimas de inserción y el sistema de garantía de ingresos y sobre su función como garante de derechos. Asegurar un derecho implica un nivel de participación ciudadano que va mucho más allá del mero asistencialismo, tan arraigado aún en nuestros días y causa, en muchos casos, del «desempoderamiento» ciudadano. Al mismo tiempo se apun- ta la necesidad de hacer valer y potenciar el marco legal existente con sus instrumentos de participación democrática, de denuncia y defensa judicial.

\section{Conclusiones, aprendizajes y retos de fu- turo}

Nuestro primer objetivo era consolidar un grupo de trabajo, estable y permanente, pero abierto siempre a la participación de otras entidades que deseen sumarse a este espacio de reflexión desde la práctica. A medio y largo plazo nos planteamos continuar por el camino hacia la ciudadanía, dando pasos hacia la constitución de comisión ciudadana, como asamblea permanente o espacio estable de reflexión y acción impulsada por y con las personas que participan en los proyectos de inserción sociolaboral, de acuerdo a sus intereses e iniciativas.

Queremos que las decisiones que se tomen sobre las acciones que se vayan a desarrollar sean acordadas por todos y surjan de la participación y el debate colectivo. Por esta razón, la incorporación de nuevas entidades y profesionales está abierta, ya que las líneas de trabajo a desarrollar por el grupo también lo están, en función de las personas que estemos participando y de las decisiones que tome el grupo.

Nuestro planteamiento y metodología de trabajo grupal, basados en el modelo de desarrollo comunitario y la investigación-acción participativa supone, en síntesis, una apuesta decidida por considerar a la persona en situación de riego o de desventaja social como sujeto protagonista de su propio proceso de crecimiento personal e inclusión social, lo que implica el reconocimiento y fortalecimiento de sus propias capacidades, como individuo y como ciudadano de pleno derecho, y la supresión de barreras que impidan el acceso a derechos tales como el trabajo, la educación, la vivienda, la igualdad de trato y oportunidades, la autonomía e independencia personal, la participación, etc.

A partir del estimulante debate generado en el grupo, los profesionales llegamos a algunas conclusiones, inquietudes e ilusiones compartidas:

- La necesidad de impulsar la participación y el asociacionismo de las personas con las que trabajamos como una línea estratégica de trabajo común y un aspecto clave dentro de su proceso de desarrollo e inserción. 
- La necesidad sistematizar una metodología sobre el desarrollo de la ciudadanía, que reúna el para qué (el sentido y finalidad de los talleres, las actividades y las asambleas que llevemos a cabo), el cómo (pautas, técnicas, estrategias) el cuándo (tiempos, plazos, ritmos) y el con qué (recursos humanos, materiales, tangibles e intangibles), etc.

- La necesidad de incorporar a las diferentes áreas de nuestras organizaciones e instituciones en este proceso participativo de promoción de la ciudadanía.

- La pertinencia de incorporar esta dinámica de talleres de ciudadanía y aprendizaje de y en, la participación, en las agendas de trabajo y procesos de autoformación de las organizaciones en las que trabajamos o colaboramos de forma voluntaria.
- La importancia de impulsar los espacios de participación ya existentes (asambleas, buzones de sugerencias, etc.) y hacerlos efectivos.

- Buscar la coherencia entre nuestras formas de organización interna y estilos de trabajo: que la participación y la horizontalidad cale también en nuestras estructuras y metodologías.

Simultáneamente, desde el Observatorio de la Exclusión y procesos de inclusión en la Comunidad de Madrid, estamos analizando nuestras metodologías de inclusión, y abriendo foros de debate sobre diferentes aspectos y temáticas (privatización de los servicios sociales, políticas activas de empleo, rentas mínimas de inserción, situación de los menores no acompañados, etc.) que inciden en nuestro trabajo y en las posibilidades de integración de muchas personas.

\section{Referencias bibliográficas}

Alonso, J., Del Rio, E. et al. (eds.) (1993). Investigación - Acción Participativa. Revista Documentación Social, 92, 59-69.

Alves, M., Basagoiti, M. y Bru, M. (coords.) (2009). De la exclusión a la ciudadanía. Análisis de los factores, barreras y oportunidades. Madrid: AMEI.

Borja, J. (febrero-mayo, 2002). La ciudad y la nueva ciudadanía. Revista Factoría, 17.

Laparra, M., Renes, V., Subirats, J. et al. (2007). Una propuesta de consenso sobre el concepto de exclusión. Implicaciones metodológicas. Revista Española del Tercer Sector, 5, 15-57.

Marshall, T. H. y Bottomore, T. (1998). Ciudadanía y clase social. Madrid: Editorial Alianza.

Martínez de Pisón, J. (1997). Derechos Humanos: su historia, su fundamento y su realidad. Zaragoza: Editorial Egido.

Max Neef, M. (2006). Desarrollo a Escala Humana. Conceptos, aplicaciones y reflexiones. ( $3^{\mathrm{a}}$ edición). Barcelona: Editorial Icaria.

Pérez Serrano, G. (1990): Investigación Acción. Aplicaciones al campo social y educativo. Madrid: Dykinson.

Raya, E. (2004). Exclusión social y Ciudadanía: claroscuros de un concepto. Aposta-Revista de Ciencias Sociales, 9, 1-18.

Rubio, M. J. y Varas, J. (1997). El análisis de la realidad en la intervención social. Métodos y técnicas de investigación. Madrid: Editorial CCS. 